\title{
Barriers to Optimal End-of-Life Care for Adolescents and Young Adults With Cancer: Bereaved Caregiver Perspectives
}

\author{
Jennifer W. Mack, MD, MPH',2,3; Erin R. Currie, PhD, RN, CPLC ; Vincent Martello, BA ${ }^{5}$; Jordan Gittzus, BS 2,6; \\ Asisa Isack, $\mathrm{BA}^{2}$; Lauren Fisher, $\mathrm{MPH}^{2}$; Lisa C. Lindley, PhD, RN; Stephanie Gilbertson-White, PhD, APRN-BC; \\ Eric Roeland, $\mathrm{MD}^{9}$; and Marie Bakitas, DNSc, CRNP ${ }^{4}$
}

\section{ABSTRACT}

Background: Adolescents and young adults (AYAs; aged 15-39 years) with cancer frequently receive intensive measures at the end of life (EoL), but the perspectives of AYAs and their family members on barriers to optimal EoL care are not well understood. Methods: We conducted qualitative interviews with 28 bereaved caregivers of AYAs with cancer who died in 2013 through 2016 after receiving treatment at 1 of 3 sites (University of Alabama at Birmingham, University of lowa, or University of California San Diego). Interviews focused on ways that EoL care could have better met the needs of the AYAs. Content analysis was performed to identify relevant themes. Results: Most participating caregivers were White and female, and nearly half had graduated from college. A total of $46 \%$ of AYAs were insured by Medicaid or other public insurance; $61 \%$ used hospice, $46 \%$ used palliative care, and $43 \%$ died at home. Caregivers noted 3 main barriers to optimal EoL care: (1) delayed or absent communication about prognosis, which in turn delayed care focused on comfort and quality of life; (2) inadequate emotional support of AYAs and caregivers, many of whom experienced distress and difficulty accepting the poor prognosis; and (3) a lack of home care models that would allow concurrent life-prolonging and palliative therapies, and consequently suboptimal supported goals of AYAs to live as long and as well as possible. Delayed or absent prognosis communication created lingering regret among some family caregivers, who lost the opportunity to support, comfort, and hold meaningful conversations with their loved ones. Conclusions: Bereaved family caregivers of AYAs with cancer noted a need for timely prognostic communication, emotional support to enhance acceptance of a poor prognosis, and care delivery models that would support both life-prolonging and palliative goals of care. Work to address these challenges offers the potential to improve the quality of EoL care for young people with cancer.

J Natl Compr Canc Netw 2021;19(5):528-533 doi: $10.6004 /$ jnccn.2020.7645

\footnotetext{
${ }^{1}$ Department of Pediatric Oncology, and ${ }^{2}$ Division of Population Sciences, Center for Outcomes and Policy Research, Dana-Farber Cancer Institute, Boston, Massachusetts; ${ }^{3}$ Division of Pediatric Hematology/Oncology, Boston Children's Hospital, Boston, Massachusetts; ${ }^{4}$ School of Nursing, University of Alabama at Birmingham, Birmingham, Alabama; ${ }^{5}$ University of New England School of Osteopathic Medicine, Biddeford, Maine; ${ }^{6}$ Tufts University School of Public Health, Boston, Massachusetts; ${ }^{7}$ University of Tennessee, Knoxville College of Nursing, Knoxville, Tennessee; ${ }^{8}$ Department of Nursing, University of lowa, lowa City, lowa; and ${ }^{9}$ Division of Palliative Care, Massachusetts General Hospital, Boston, Massachusetts.
}

\section{Background}

More than 70,000 adolescents and young adults (AYAs; aged 15-39 years) are diagnosed with cancer in the United States each year, and cancer is their leading disease-related cause of death. ${ }^{1,2}$ Existing standards for care of children, adolescents, and young adults promote attention to symptoms and suffering, open communication about the future, inclusion in decisions, and support of enduring personal connections. ${ }^{3-5}$ Although young people may arrive at different decisions for the care they wish to receive, this type of support can ensure that care is reflective of personal values. ${ }^{4,6-11}$ Work to improve end-of-life (EoL) care among AYAs has therefore often focused on personal identification of values and preferences and communication of preferences within the family. ${ }^{9,10,12,13}$

However, even with increasing efforts to meet the needs of AYAs with advanced cancer, this population frequently receives intensive measures at EoL, including late-life hospitalizations, chemotherapy, and care in the ICU. ${ }^{14-16}$ Many die in the hospital, and hospice services are underutilized to support those who die at home, especially among minority patients and those of lower socioeconomic status. ${ }^{16,17}$ We do not know whether such care reflects the personal preferences of AYAs or represents broader challenges requiring ongoing efforts to improve care quality for this young population.

To understand these issues in more depth, we sought to identify barriers to optimal EoL care for AYAs. We interviewed bereaved family caregivers of AYAs who died of cancer about their experiences with care. Semistructured interviews were conducted with caregivers from 3 healthcare centers: University of Alabama at Birmingham, University of Iowa, and University of California San Diego. Caregivers were asked to reflect on EoL care, including any ways that it could have better met the needs of AYAs. We specifically assessed barriers to the use of palliative care, hospice, and home death to understand the extent to which choices were based on values or, alternatively, were driven by logistical and other challenges 
that could be remediable with intervention. For this study, we defined palliative care as an approach to care focused on improving quality of life for patients with lifethreatening illness, and defined hospice as a type of healthcare, often provided in the home but sometimes also available as an inpatient experience, that is focused on treating pain, symptoms, and emotional and spiritual distress for those nearing EoL.

\section{Methods}

\section{Design, Sample, and Setting}

This was a qualitative interview study of bereaved family caregivers of AYAs who died of cancer. Qualitative research methods were selected to allow an exploration of caregiver perspectives. We conducted semistructured interviews by phone in English or Spanish as part of a larger study at the 3 healthcare centers, which are Palliative Care Research Cooperative Group member sites. We identified 252 AYAs aged 15 to 39 years who died of cancer in 2013 through 2016 and reviewed medical records to examine care received at EoL as part of the larger study. ${ }^{18}$ Bereaved caregivers, identified in medical records, were then contacted by mail with a letter inviting participation in a survey and in-depth interview by phone. One caregiver per patient was eligible if he or she spoke English or Spanish and was a self-identified primary informal caregiver of the AYA patient. When more than one caregiver was listed in the medical record, each was contacted sequentially to ensure that only one caregiver per patient participated. Signed informed consent was required for participation. The Institutional Review Boards of all involved sites approved this study.

\section{Data Collection}

Interviews were conducted from June 2017 to July 2018. We planned to interview 15 to 30 caregivers based on general recommendations for qualitative research, ${ }^{19}$ with a goal of continuing interviews until we reached thematic saturation. To identify caregivers across socioeconomic status categories, we specifically sought caregivers of AYAs who were uninsured, insured by Medicaid, or who lived in a zip code with a median income $\leq 200 \%$ of the federal poverty level (FPL). Overall, 260 caregivers of the 252 patients were identified for the parent study through medical records. Of those, 80 could not be reached because of an incorrect or changed address, 66 declined to participate, and 74 did not respond. Of the 40 who agreed to participate, 35 completed surveys and 28 completed in-depth interviews; because we reached thematic saturation at 28 participants, no further participation was sought.

Trained research assistants (V. Martello, J. Gittzus, A. Isack) conducted semistructured interviews by phone in English or Spanish using a semistructured interview guide. Questions focused on decisions made about the use of hospice, palliative care, and location of death; ways in which care did or did not meet the needs of the AYAs; and barriers to best EoL care (Table 1). Probing questions were used to encourage reflection. Interviews were audiotaped, transcribed, and verified for accuracy.

\section{Data Analysis}

Content analysis was performed on coded texts to identify themes and variations, reflecting themes shown in Table $1 .{ }^{19}$ We developed an initial codebook based on a priori hypotheses about care decisions and possible barriers to high-quality EoL care. Additional themes were incorporated into the codebook as they arose. Three authors (V. Martello, A. Isack, J.W. Mack) participated in coding, with each transcript coded by 2 to 3 coders. Coding was performed independently; coders then met to discuss codes and reconcile findings when needed. Although coding was entirely performed by human coders, NVivo software (QSR International, Burlington, $\mathrm{NH}$ ) was used to annotate themes within interview texts and to perform cross-checks of related themes.

\section{Results}

Of 28 participating caregivers, three-quarters were female (Table 2). Half were bereaved parents of the AYAs, whereas $40 \%$ were spouses or partners. One-quarter of the AYA patients were aged 15 to 21 years at death, $32 \%$ were aged 22 to 29 years, and $43 \%$ were aged 30 to 39 years (Table 3 ). Nearly half (46\%) had Medicaid or other public insurance, and $54 \%$ lived in a low-income zip code (median income $\leq 200 \%$ FPL). Nearly half of caregivers were college graduates $(46 \%)$.

Participating caregivers reported that 17 patients received home hospice services, 13 received palliative care consultations, and fewer than half $(n=12)$ died at home. Of the AYAs who died at home, 5 were in the youngest age range (15-21 years) and 7 of their participating caregivers were parents. Caregivers described several priorities as they made EoL decisions, including a desire for the patient to experience physical comfort and emotional support. EoL decisions were not typically focused on the specific location of care or services used, and not every caregiver could recall whether their AYAs had used palliative care or hospice. Rather, caregivers sought care that aligned with the goals of the AYAs and supported the family, and the use of hospice, palliative care, and either inpatient or home death were welcomed if they could support the goals of the AYAs.

Despite a clear sense of these priorities, many caregivers experienced lingering questions or regrets about the care that was received, especially when they felt that comfort and emotional support were not 


\section{Table 1. In-Depth Interview Themes and Questions}

\begin{tabular}{|ll|}
\hline Theme & Question \\
\hline Prognostic awareness & $\begin{array}{l}\text { I'd like to start by hearing about when you first } \\
\text { realized that [patient] was not likely to survive. }\end{array}$ \\
$\begin{array}{l}\text { Use of hospice and } \\
\text { palliative care }\end{array}$ & $\begin{array}{l}\text { Some patients and families want to use } \\
\text { supports like hospice and palliative care. [Offer } \\
\text { definitions if needed.] Do you know if [patient] } \\
\text { wished to receive hospice? What about } \\
\text { palliative care? What were your own wishes? }\end{array}$ \\
\hline $\begin{array}{l}\text { Decisions about hospice } \\
\text { and palliative care }\end{array}$ & $\begin{array}{l}\text { What did you and [patient] decide about } \\
\text { hospice? What about palliative care? Why did } \\
\text { you and [patient] make those decisions? }\end{array}$ \\
\hline Location of death & $\begin{array}{l}\text { Some patients have specific wishes about } \\
\text { where they want to die. Did [patient] have } \\
\text { wishes about where he or she would die? What } \\
\text { were your own wishes? }\end{array}$ \\
\hline $\begin{array}{l}\text { Decisions about location } \\
\text { of death }\end{array}$ & $\begin{array}{l}\text { What did you and [patient] decide about where } \\
\text { he or she would die? Why did you and [patient] } \\
\text { make those decisions? }\end{array}$ \\
\hline Other decisions & $\begin{array}{l}\text { Were there other important decisions that you } \\
\text { or [patient] made about how care would go at } \\
\text { the end of life? What were those decisions? }\end{array}$ \\
\hline Barriers to care & $\begin{array}{l}\text { Did anything stand in the way of getting the } \\
\text { care that you and [patient] wanted? What were } \\
\text { those barriers? }\end{array}$ \\
\hline Quality of care & $\begin{array}{l}\text { All in all, how did care go at the end of life for } \\
\text { [patient]? Was anything more difficult than it } \\
\text { had to be? In retrospect, what kind of support } \\
\text { would have been ideal for [patient]? Why? }\end{array}$ \\
\hline
\end{tabular}

Questions are shown in brief; probing questions and explanatory text are not shown.

optimal. Caregivers noted 3 main barriers to optimal EoL care for their AYAs: suboptimal communication, emotional challenges, and inflexible or limited care options.

\section{Communication Barriers}

Suboptimal communication about a patient's poor prognosis was emphasized by 12 caregivers as a barrier to optimal EoL care. When prognosis communication occurred late in the patient's illness, caregivers felt that they missed the opportunity to make timely decisions about care focused on comfort and quality of life. As a caregiver said, "I think we waited way too long. I think he suffered in pain much longer than he should have.... I was not aware of how close he was to death, and I think that is the one thing I wish I would have known. Perhaps we could have provided comfort much earlier than we did." Another caregiver stated, "I feel I didn't have a lot of information. I think maybe knowing all along where he was might have helped make some better decisions... and just trying to keep him comfortable...if that's where we were, we should have addressed that rather than trying to continue treatment that...was not going to change anything."

Sometimes communication about prognosis did not take place at all, and caregiver understanding of the patient's health status often came from inference rather

\section{Table 2. AYA Caregiver Characteristics}

\begin{tabular}{|c|c|}
\hline Characteristic & n (\%) \\
\hline Caregivers, $\mathrm{N}$ & 28 \\
\hline \multicolumn{2}{|l|}{ Sex } \\
\hline Male & $7(25)$ \\
\hline Female & $21(75)$ \\
\hline \multicolumn{2}{|l|}{ Ethnicity } \\
\hline Hispanic & $3(11)$ \\
\hline Non-Hispanic & 25 (89) \\
\hline \multicolumn{2}{|l|}{ Race } \\
\hline White & $23(82)$ \\
\hline Black & $2(7)$ \\
\hline Asian/Pacific Islander & $2(7)$ \\
\hline Other & $1(4)$ \\
\hline \multicolumn{2}{|l|}{ Highest level of school } \\
\hline$<$ High school & $2(7)$ \\
\hline High school graduate & $10(36)$ \\
\hline Technical school or associates degree & $3(11)$ \\
\hline College graduate & $6(21)$ \\
\hline Advanced degree & $7(25)$ \\
\hline \multicolumn{2}{|l|}{ Relationship to patient } \\
\hline Spouse & $10(36)$ \\
\hline Partner & $1(4)$ \\
\hline Child & $1(4)$ \\
\hline Parent & $14(50)$ \\
\hline Other relative & $2(7)$ \\
\hline \multicolumn{2}{|l|}{ Religion } \\
\hline Catholic & $5(18)$ \\
\hline Protestant/Other Christian & $17(61)$ \\
\hline Jewish & $0(0)$ \\
\hline Other & $2(7)$ \\
\hline No religion & $4(14)$ \\
\hline
\end{tabular}

Abbreviation: AYA, adolescent and young adult.

than direct conversation: "He realized-actually, we realized - that he was not probably going to make it when his doctor wouldn't give us no kind of information." In addition to affecting care decisions, late or absent communication took away opportunity for conversations with the patient: "I just wish in this case those doctors had been clear with me, because I needed a little more time to say goodbye than what I did." Missed opportunity for these discussions was a source of lingering regret.

\section{Emotional Barriers}

Even when information was provided, patients and caregivers sometimes had difficulty accepting the patient's poor prognosis. As one caregiver admitted, "If anything stood 


\section{Table 3. AYA Patient Characteristics}

\begin{tabular}{|c|c|}
\hline Characteristic & n (\%) \\
\hline Patients, N & 28 \\
\hline \multicolumn{2}{|l|}{ Sex } \\
\hline Male & $16(57)$ \\
\hline Female & $12(43)$ \\
\hline \multicolumn{2}{|l|}{ Ethnicity } \\
\hline Hispanic & $3(11)$ \\
\hline Non-Hispanic & $24(86)$ \\
\hline Unknown & $1(4)$ \\
\hline \multicolumn{2}{|l|}{ Race } \\
\hline White & $21(75)$ \\
\hline Black & $3(11)$ \\
\hline Asian/Pacific Islander & $1(4)$ \\
\hline Other & $0(0)$ \\
\hline Unknown & $3(11)$ \\
\hline \multicolumn{2}{|l|}{ Age at death, y } \\
\hline $15-21$ & $7(25)$ \\
\hline $22-29$ & $9(32)$ \\
\hline $30-39$ & $12(43)$ \\
\hline \multicolumn{2}{|l|}{ Insurance ${ }^{a}$} \\
\hline Private & $14(50)$ \\
\hline Medicaid/Public & $13(46)$ \\
\hline Uninsured & $0(0)$ \\
\hline Other & $1(4)$ \\
\hline Unknown & $1(4)$ \\
\hline \multicolumn{2}{|l|}{ Median income by zip code of residence } \\
\hline$\leq 200 \% \mathrm{FPL}$ & $15(54)$ \\
\hline$>200 \% \mathrm{FPL}$ & $13(46)$ \\
\hline \multicolumn{2}{|l|}{ Site of care } \\
\hline University of California San Diego & $10(36)$ \\
\hline University of lowa & $10(36)$ \\
\hline University of Alabama at Birmingham & $8(29)$ \\
\hline
\end{tabular}

Abbreviations: AYA, adolescent and young adult; FPL, federal poverty level. a One patient was dually insured by Medicaid and private insurance; numbers therefore total to 29 .

in the way [of the best EoL care], it was us just not wanting to accept the reality of what was going on." Seven caregivers commented that the patient would have benefited from emotional support in coming to terms with the illness and dying. One caregiver said, "I guess somebody could have talked to him about being afraid, realizing that he wasn't coming home, because I think he had figured that out at that point." Eight caregivers also noted a need for better emotional support of the caregiver and a need for open conversations within the family. One caregiver stated, "I had a hard time accepting [her prognosis] and everything, and I really wish I would have talked to her more about life without her. Maybe, I don't know, maybe some type of counseling to just get me to talk to [her]." Emotional barriers sometimes delayed the integration of palliative care and transitions to hospice, leading patients and caregivers to instead focus on a continued search for life-prolonging measures. As with concerns about prognosis communication, these concerns contributed to lingering distress for caregivers.

In addition, 2 caregivers raised concerns about how and when conversations about prognosis and EoL care were conducted, feeling that these conversations were not always supportive of the AYA's needs. As a caregiver said, "You have to make sure people are ready for those conversations. And again, it wasn't that [she] was unaware or that any of us were unaware [of] the gravity of her diagnosis and her prognosis. It's just that we chose to handle it in a different way. You know, [she] wanted to be positive and hopeful, and that's how she coped with it."

Finally, several caregivers noted that although aspects of EoL care may have gone well, the death of a young person is fraught for both patients and caregivers. As one caregiver explained, "There's no medicine that you can give a person to help them cope with the fact that their biological existence is coming to an end. And so I think it's that existential crisis, in a way, that is the most destructive.... And I think perhaps the most depressing and awful part about watching someone die, or being with them when they die, is, you know, watching them come to terms that there is no future and then trying to live a life with no future.... And I think that's [the] hardest part about [her] death, which for me was to watch her go through that." Another caregiver put it simply: "I mean just the whole thing was difficult. It just was."

\section{Inflexible or Limited Care Options}

Several caregivers had a vision for best EoL care, but it was not always possible to achieve this vision because of limited available care options; this situation often occurred because of a desire to meet dual care goals, focused on both comfort and prolonging life, which were sometimes at odds with available care models. For example, 7 caregivers reported that the patient would have preferred to die at home but declined hospice because of issues with the care delivery model, such as an inability to receive transfusions or other life-prolonging treatments while on hospice. One caregiver commented, "They said...he couldn't have a blood transfusion because that would just extend his life. Well, we were trying to extend his life. And...the social worker kept saying to us, well that can't be paid for, that can't be paid for. You know, my husband and I both said we'll pay for it out of our own pocket if we have to." 


\section{Discussion}

We interviewed caregivers of AYAs who died of cancer to understand their experiences with care and treatment decisions near EoL. Although our interviews focused in part on specific care decisions, such as decisions about palliative care and hospice, most caregivers focused on whether care provided comfort and emotional support to their dying loved one, regardless of the care delivery model. Their experiences can help guide best practices for the care of AYAs with advanced cancer in 3 main areas.

First, several caregivers noted that their lack of knowledge of the patient's prognosis prevented them from focusing on comfort and quality of life at EoL. With many patients, clinicians provided overly optimistic information or avoided conversations entirely; as a result, caregivers were sometimes left to rely on intuition to understand what was ahead. Previous work has shown that clinicians worry that frank discussions about prognosis will cause distress and take away hope. ${ }^{20-24}$ With respect to the care of AYAs, these concerns may be heightened given the potential for emotional vulnerability among these young patients. Indeed, 2 caregivers raised concerns that conversations were not optimally sensitive to the needs of the AYAs, highlighting the need for clinicians to work with patients and families to determine the most supportive ways to communicate. However, previous work involving AYAs with cancer has also found that most AYAs want to know about their prognosis, and honest discussions about what is ahead can have positive psychosocial outcomes, including relief of distress and improved peace of mind. ${ }^{25}$ Our findings support the need for clinicians to have prognostic discussions as a prelude to effective EoL decision-making.

Second, caregivers highlighted the emotional distress that they and the AYAs experienced during these times. Many caregivers continued to experience significant distress after the young person's death. Caregivers especially noted the need for communication within the family about what was ahead and suggested that clinicians should consider facilitating these conversations. In addition, caregivers felt that AYAs desired support and assistance in coming to terms with their future or, as described by one caregiver, "a life with no future."

Finally, caregivers emphasized the practical and logistical barriers to best care. A frequent concern was a lack of robust concurrent care models to allow patients to receive both home hospice and life-prolonging therapies, such as transfusions. The 2010 Patient Protection and Affordable Care Act, which was in place during this study's time frame, offers concurrent care for patients aged $<21$ years with life-threatening illness who are insured by Medicaid/Children's Health Insurance Program (CHIP). ${ }^{26-28}$ However, adult patients (aged $\geq 21$ years) are not covered by this provision, and even for young patients, its implementation can vary. ${ }^{29}$ As a direct result of barriers to concurrent care, one-quarter of caregivers in this study reported that they opted for in-hospital death. Enhancing flexibility in care models could create an expanded array of options for young patients whose needs may differ from those of older patients.

These findings also highlight that many AYAs with advanced cancer have more than one goal of care. Although caregivers reported that many AYAs wanted comfort and quality of life, they also often wanted to live as long as possible. Interventions such as transfusions were seen as supporting a desire to continue to live the best and longest life possible. Regardless of care setting, clinicians should be aware that young patients will not always hold a single, most important care goal. Instead, supporting a spectrum of needs may be required for best care. ${ }^{6-8,12,30}$

We sought to include caregivers of patients with diverse socioeconomic backgrounds given the potential for special challenges to EoL care delivery for patients with limited resources. However, although nearly half of our sample was insured by Medicaid and half lived in low-income areas, most participating caregivers were White women and nearly half were college educated. Therefore, diversity was not optimal despite our efforts to explore the challenges of a more resource-limited population. Perhaps as a result, identified themes generally reflected challenges that have been observed broadly in other populations. However, prior work has found especially high rates of inpatient deaths and low hospice use among AYAs living in poverty. ${ }^{16,17}$ It is possible that suboptimal communication and challenges with hospice care delivery models are experienced disproportionately by AYAs with limited resources. Further evaluation in future work with a more diverse population is needed.

Our study has some important limitations. In addition to limited diversity, this was a substudy of 28 family caregivers within the larger parent trial that reviewed the medical records of 252 AYAs. Many caregivers could not be reached because of changes of address, and others declined participation. Thus, we cannot be certain that our participants' views accurately reflect those of the wider population. Quantitative work is needed to ensure that findings are generalizable. Nonetheless, this study also has notable strengths, including the participation of caregivers from 3 healthcare sites. Rigorous qualitative research methods also allowed us to explore perspectives on care and begin to address an important gap in the knowledge base.

\section{Conclusions}

Our findings suggest that high rates of intensive EoL care and inpatient deaths among AYAs may not uniformly reflect a desire to pursue all possible options in this young population. Instead, caregivers feel that communication 
and care delivery may not always meet AYAs' needs to feel as good as possible and live as long as possible at EoL. Future research and care models should enhance efforts to help AYAs understand their prognoses and live their remaining days in the best way possible.

Submitted June 18, 2020; accepted for publication August 27, 2020

Published online February 11, 2021.

Author contributions: Study concept: Mack. Data collection: All authors. Data analysis: Mack, Martello, Gittzus, Isack, Fisher. Writing - original draft: Mack. Writing - review and editing: Currie, Martello, Gittzus, Isack, Fisher,

Lindley, Gilbertson-White, Roeland, Bakitas.
Disclosures: The authors have not received any financial consideration from any person or organization to support the preparation, analysis, results, or discussion of this article.

Funding: Research reported in this article was supported by the NIH under award number R21 R21NR016580 (Dr. Mack) and by the Palliative Care Research Cooperative Group, funded by the National Institute of Nursing Research (U2CNR014637). Dr. Currie is supported by the National Palliative Care Research Center. Dr. Roeland is sponsored by the Cambia Health Foundation Sojourns Scholar Award.

Disclaimer: The content is solely the responsibility of the authors and does not necessarily represent the official views of the $\mathrm{NIH}$.

Correspondence: Jennifer W. Mack, MD, MPH, Department of Pediatric Oncology, Dana-Farber Cancer Institute, 450 Brookline Avenue, Boston, MA 02215. Email: Jennifer_mack@dfci.harvard.edu

\section{References}

1. Bleyer A. The adolescent and young adult gap in cancer care and outcome. Curr Probl Pediatr Adolesc Health Care 2005;35:182-217.

2. Bleyer A, O'Leary M, Barr R, eds. Cancer Epidemiology in Older Adolescents and Young Adults 15 to 29 Years of Age, Including SEER Incidence and Survival: 1975-2000. NIH Pub. No. 06-5767. Bethesda, MD: National Cancer Institute; 2006.

3. Weaver MS, Heinze KE, Bell CJ, et al. Establishing psychosocial palliative care standards for children and adolescents with cancer and their families: an integrative review. Palliat Med 2016;30:212-223.

4. Sansom-Daly UM, Wakefield CE, Patterson $P$, et al. End-of-life communication needs for adolescents and young adults with cancer: recommendations for research and practice. J Adolesc Young Adult Oncol 2020;9:157-165.

5. Weaver MS, Heinze KE, Kelly KP, et al. Palliative care as a standard of care in pediatric oncology. Pediatr Blood Cancer 2015;62(Suppl 5):S829-833.

6. Wein S, Pery S, Zer A. Role of palliative care in adolescent and young adult oncology. J Clin Oncol 2010;28:4819-4824.

7. Pritchard S, Cuvelier G, Harlos M, et al. Palliative care in adolescents and young adults with cancer. Cancer 2011;117(10 Suppl):2323-2328.

8. Hinds PS, Drew D, Oakes LL, et al. End-of-life care preferences of pediatric patients with cancer. J Clin Oncol 2005;23:9146-9154.

9. Wiener L, Ballard E, Brennan T, et al. How I wish to be remembered: the use of an advance care planning document in adolescent and young adult populations. J Palliat Med 2008;11:1309-1313.

10. Lyon ME, Jacobs $S$, Briggs $L$, et al. Family-centered advance care planning for teens with cancer. JAMA Pediatr 2013;167:460-467.

11. Lyon ME, Garvie PA, McCarter R, et al. Who will speak for me? Improving end-of-life decision-making for adolescents with HIV and their families. Pediatrics 2009;123:e199-206.

12. Wiener $L$, Zadeh $S$, Battles $H$, et al. Allowing adolescents and young adults to plan their end-of-life care. Pediatrics 2012;130:897-905.

13. Friebert $\mathrm{S}, \mathrm{Grossoehme} \mathrm{DH}$, Baker JN, et al. Congruence gaps between adolescents with cancer and their families regarding values, goals, and beliefs about end-of-life care. JAMA Netw Open 2020;3:e205424.

14. Johnston EE, Alvarez E, Saynina O, et al. End-of-life intensity for adolescents and young adults with cancer: a Californian population-based study that shows disparities. J Oncol Pract 2017;13:e770-781.

15. Mack JW, Chen LH, Cannavale K, et al. End-of-life care intensity among adolescent and young adult patients with cancer in Kaiser Permanente Southern California. JAMA Oncol 2015;1:592-600
16. Mack JW, Chen K, Boscoe FP, et al. High intensity of end-of-life care among adolescent and young adult cancer patients in the New York State Medicaid program. Med Care 2015;53:1018-1026.

17. Johnston EE, Bogetz J, Saynina O, et al. Disparities in inpatient intensity of end-of-life care for complex chronic conditions. Pediatrics 2019;143: e20182228

18. Roeland EJ, Lindley LC, Gilbertson-White S, et al. End-of-life care among adolescent and young adult patients with cancer living in poverty. Cancer 2020;126:886-893.

19. Krippendorff K. Content Analysis: An Introduction to Its Methodology, 2nd ed. Newbury Park, CA:SAGE Publications; 2004.

20. Ruddick W. Hope and deception. Bioethics 1999;13:343-357.

21. Kodish E, Post SG. Oncology and hope. J Clin Oncol 1995;13:1817.

22. Baile WF, Lenzi R, Parker PA, et al. Oncologists' attitudes toward and practices in giving bad news: an exploratory study. J Clin Oncol 2002;20: 2189-2196.

23. Delvecchio Good MJ, Good BJ, Schaffer C, et al. American oncology and the discourse on hope. Cult Med Psychiatry 1990;14:59-79.

24. Lamont EB, Christakis NA. Prognostic disclosure to patients with cancer near the end of life. Ann Intern Med 2001;134:1096-1105.

25. Mack JW, Fasciano KM, Block SD. Communication about prognosis with adolescent and young adult patients with cancer: information needs, prognostic awareness, and outcomes of disclosure. J Clin Oncol 2018;36: 1861-1867.

26. Lindley LC, Keim-Malpass J, Svynarenko R, et al. Pediatric concurrent hospice care: a scoping review and directions for future nursing research. J Hosp Palliat Nurs 2020;22:238-245.

27. Lindley LC. Health care reform and concurrent curative care for terminally ill children: a policy analysis. J Hosp Palliat Nurs 2011 13:81-88.

28. Mooney-Doyle K, Keim-Malpass J, Lindley LC. The ethics of concurrent care for children: a social justice perspective. Nurs Ethics 2019;26: 1518-1527.

29. Lindley LC, Edwards S, Bruce DJ. Factors influencing the implementation of health care reform: an examination of the Concurrent Care for Children provision. Am J Hosp Palliat Care 2014;31:527-533.

30. Walter JK, Rosenberg AR, Feudtner C. Tackling taboo topics: how to have effective advanced care planning discussions with adolescents and young adults with cancer. JAMA Pediatr 2013;167:489-490. 